\title{
Accurate source localization approach based on illuminating LEDs in indoor environment
}

\author{
Yan Zhang ${ }^{1, \mathrm{a}}$, Xiansheng Guo, ${ }^{2, \mathrm{~b}}$, and Ya Zhou ${ }^{3, \mathrm{c}}$ \\ ${ }^{1}$ School of Architecture and Civil Engineering, Chengdu University, Chengdu, China \\ ${ }^{2}$ School of Electronic Engineering, University of Electronic Science and Technology of China, \\ Chengdu, China \\ ${ }^{3}$ Architecture and Art of College, North China University of Technology, Beijing, China \\ a39624001@qq.com, bxsguo@uestc.edu.cn, c736272872@qq.com
}

Keywords: Received signal strength, LED, Visible Light Communications, indoor localization.

\begin{abstract}
We propose a new positioning system based on the received signal strength (RSS) fingerprinting approach. The RSSs of LEDs are influenced by some ambient parameters, such as radiation pattern, incidence angle, attenuation coefficient, multipath fading and so on. And these factors degrade the performance of some existing range-based localization approaches significantly. To overcome this drawback, we prestore the RSS measurements of different grids by offline training phase. In the online localization phase, we compare the instantaneous RSS with the prestored RSS measurements using correlation matching technique. Our proposed approach can improve the accuracy of localization significantly without calibration process. The simulation results show the efficacy of our approach.
\end{abstract}

\section{Introduction}

White light emitting diodes (LEDs) have been widely used because of lighting efficiency, eco friendliness, and lifetime. The visible light communication (VLC) based on LEDs can be used as lighting system and communication system simultaneously. Indoor localization system based on visible light communication has many advantages including low cost, high data rate, and high accuracy. For such reasons, indoor positioning systems using VLC have recently gained popularity as effective alternatives [1].

Many approaches have been proposed for indoor localization using LEDs including TOA-based [2], TDOA-based [3], AOA-based [4], and mixed-based localization approaches [5]. All the listed approaches decrease the errors of localization to some extent. However, most of them need calibration of received power or some auxiliary devices in their localization process. Comparatively speaking, the localization technique based on RSS measurements of LEDs can be easily implemented and it has low cost with slightly low accuracy [6]. Some existing RSS-based approaches need calibrate the received power to improve the estimate accuracy of range [1]. The calibration process not only makes the localization system impractical in practice but also brings other errors to the localization results.

Additionally, the RSSs of LEDs are influenced by some ambient parameters, such as radiation pattern, incidence angle, attenuation coefficient, multipath fading and so on. And these factors degrade the performance of some existing range-based localization approaches significantly. To avoid the calibration process, we present a RSS fingerprinting based localization approach to improve the accuracy of indoor localization system. The steps of our approach are as follows: firstly, we prestore the RSS measurements of different grids by offline training phase; sencondly, in the online localization phase, we compare the instantaneous RSS with the prestored RSS measurements using correlation matching technique. And the estimates of target nodes are given by searching the maximum value of correlation coefficient. Our proposed approach can improve the accuracy of localization significantly without calibration process. The simulation results show the efficacy of our approach. 


\section{Related Work}

The received power $P_{r}$ in a light-of-sight environment can be expressed as

$$
P_{r}=\frac{P_{t}}{d^{2}} R(\phi) I(\psi)
$$

where $P_{t}$ is the average transmitted optical power, $d$ is the distance between the transmitter and receiver, and $\phi$ and $\psi$ are radiation and incidence angles with respect to the transmitter and receiver, respectively. Transmitter radiant intensity $R(\phi)$ and effective signal collection area $I(\psi)$ can be written as

$$
\begin{aligned}
& R(\phi)=\frac{m+1}{2 \pi} \cos ^{m} \phi \\
& I(\psi)=A \cos \psi T_{g}(\psi) T_{c}(\psi)
\end{aligned}
$$

where $m=-\ln 2 / \ln \left(\cos \psi_{1 / 2}\right)$ is the order with $\psi_{1 / 2}$ being the transmitter semi-angle. $A, T_{g}(\psi)$, and $T_{c}(\psi)$ are physical area of detector, gain of the optical filter, and concentrator gain, respectively. From Equations (1), (2), and (3), we know the distance $d$ may be given by

$$
\tilde{d}=\sqrt{\frac{P_{t}}{P_{r}} \frac{m+1}{2 \pi} \cos ^{m} \phi A \cos \psi T_{g}(\psi) T_{c}(\psi)}
$$

Obviously, it is a difficult task to estimate the distance $d$ between transmitter and receiver accurately from $P_{r}$ because the received power is influenced by several factors. The current of the photo detector, $i_{P D}$, at the receiver is generated in proportion to the received optical power with a certain sensitivity, to simplify the expression of $\tilde{d}$, we have

$$
\tilde{d}=\left(\frac{C_{R F}}{P_{R F}}\right)^{1 / 4},
$$

where $P_{R F}$ is the RF power received from receiver and $C_{R F}$ is the RF power constant related to the optical-to-electrical conversion efficiency. The $P_{R F}$ means the received power under normal conditions, which refers to when only the normal radiation and incidence are considered. The RF power satisfies $\left(P_{R F}\right)^{1 / 2} \propto i_{P D} \propto P_{r}$. In a real VLC link, the received RF power is affected by the radiation and incidence angle. Hence, $(5)$ can be modified as

$$
P_{R F}=\left(C_{R F} / \bar{d}^{4}\right) G_{r}(\phi) G_{i}(\psi),
$$

where $G_{r}(\phi)$ and $G_{i}(\psi)$ are the normalized radiation and incidence gains of a transmitter and a receiver with respect to $\phi$ and $\psi$, respectively. The characteristics of $G_{r}(\phi)$ and $G_{i}(\psi)$ can be modeled as Lambertian property as follows

$$
G_{r}(\phi)=\cos ^{m_{r}} \phi, G_{i}(\psi)=\cos ^{m_{i}} \psi
$$

where $m_{r}=-\ln 2 / \ln \left(\cos \phi_{1 / 2}\right), m_{i}=-\ln 2 / \ln \left(\cos \psi_{1 / 2}\right)$. It is worthy to point out that the optical attenuation factors are not considered in (5). In general, the range estimate is larger than the real range. Hence, a weighted calibration method is proposed to calibrate the estimated range as follow [1]

$$
\hat{d}=W \times \tilde{d}
$$

where $W=\left(C_{n} / \tilde{d}\right)^{n}, 0 \leq n<1$ with $C_{n}$ being a normalizing postive constant. In order to obtain a more accurate estimate of $\hat{d}$, one needs to search a optimal $n$ in the region of $0 \leq n<1$ using some optimization techniques. 
Consider that we have $M$ transmitters with known coordinates $\boldsymbol{p}_{i}=\left[x_{i}, y_{i}\right]^{T}(i=1,2, \cdots, M)$, and the unknown coordinate of receiver is $\boldsymbol{O}=[x, y]^{T}$. Using (8), we can obtain the multiple estimates of range $d_{i},(i=1,2, \cdots, M)$ and the following equations hold

$$
\left\{\begin{array}{c}
\left(x-x_{1}\right)^{2}+\left(y-y_{1}\right)^{2}=\hat{d}_{1}^{2} \\
\left(x-x_{2}\right)^{2}+\left(y-y_{2}\right)^{2}=\hat{d}_{2}^{2} \\
\vdots \\
\left(x-x_{M}\right)^{2}+\left(y-y_{M}\right)^{2}=\hat{d}_{M}^{2}
\end{array}\right.
$$

The matrix form of (9) can be expressed as

$$
A \boldsymbol{o}=\boldsymbol{b}
$$

The location estimate of receiver can be solved from (10) using linear least squares (LLS) [7], weighted least squares (WLS) [8], or two-step weighted least squares (2WLS) techniques [9], and so on.

\section{The proposed approach}

Fingerprinting-based approach Fingerprinting-based localization for LED in indoor environment is a new and very active field currently. The key idea of fingerprinting is that each location has a set of unique features, and this set of features or the "print" will be used to identify a specific location. In order to use this methodology, we should divide the indoor environment into $N$ grids based on the requirement of localization accuracy. Then the process of RSS fingerprinting can be divided into the following primary phases:

1. Offline Training Phase: For each of $N$ positions $\left\{x_{j}, y_{j}\right\}_{j=1}^{N}$ (on a grid) in the area of interest, signal measurements are taken from $M$ transmitters to produce the associated RSS fingerprints $\boldsymbol{F}_{j}=\left[\left(x_{j}, y_{j}\right), \boldsymbol{s}_{j}\right]_{j=1}^{N}$, where $\boldsymbol{s}_{j}=\left[s_{j 1}, \cdots, s_{j M}\right]$. The observation data $s_{j}$ is the received power $P_{r}$ from (1). That is to say, we use the original received power without any calibration process. Then the fingerprints are collected to construct a "radio map", which is stored in a database prior to the localization process. We denote the database as $\mathcal{F}=\left[\boldsymbol{F}_{1}^{T}, \boldsymbol{F}_{2}^{T}, \cdots, \boldsymbol{F}_{N}^{T}\right]$.

2. Online Localization Phase: Assuming that the receiver locates in grid $i$. Upon arrival of a signal, the received power at this point can be given by $\left[\left(x_{i}, y_{i}\right), \overline{\boldsymbol{s}}_{i}\right]$. The received power of interest $\overline{\boldsymbol{s}}_{i}=$ $\left[\bar{s}_{i 1}, \cdots, \bar{s}_{i M}\right]$ is extracted and compared to the radio map using one or more pattern matching techniques (e.g., $k$-nearest neighbours, correlation matching or distance minimization, etc.).

3. Correlation matching technique: We consider the correlation matching technique in the selection process. The receiver location is estimated as $\left[\hat{x}_{i}, \hat{y}_{i}\right]$ by selecting the best match grid point in the "radio map". The correlation coefficient is computed by

$$
r_{j}=\frac{\overline{\boldsymbol{s}}_{i} \overline{\boldsymbol{s}}_{j}^{T}}{\left\|\overline{\boldsymbol{s}}_{i} \overline{\boldsymbol{s}}_{j}^{T}\right\|}
$$

The maximal index can be found by maximizing the coefficient vector $\boldsymbol{r}=\left[r_{1}, r_{2}, \cdots, r_{N}\right]$

$$
t=\arg \max _{1 \leq t \leq N} \mathbf{r}
$$

The location estimate of receiver is given by $(\hat{x}, \hat{y})=\boldsymbol{F}_{t}(1: 2)$. 


\section{Simulation Results}

The interesting space has dimensions of $60 \mathrm{~cm} \times 60 \mathrm{~cm} \times 60 \mathrm{~cm}$. The transmitters consist of 9 LEDs with coordinates $(0,0,60),(30,0,60),(60,0,60),(0,30,60),(30,30,60),(60,30,60),(0,60,60)$, $(30,60,60)$ and $(60,60,60)$. In order to apply VLC to indoor localization without inter-cell interference, we transmitted different carriers in different transmitters, we generated four $0.25-\mathrm{Ms} / \mathrm{s}$ QPSK signals with $2 \mathrm{MHz}, 2.5 \mathrm{MHz}, 3 \mathrm{MHz}$, and $3.5 \mathrm{MHz}$ carriers for the four different transmitters. The parameters are listed as follows. In (5), $C_{R F}=7.8 \times 10^{3}\left[\mathrm{~cm}^{4} \cdot \mathrm{mW}\right]$ and the calibration factor $n=0.7286$ in (8) were used in our experiment. The normalized constant is set to 60. In the offline training phase, we assumed that the receiver was located at the ground and we made a grid with equal spacing of $3 \mathrm{~cm}$. We placed the receiver at each point on the grid and recorded the received power from the four transmitters to construct a database $\mathcal{F}$. Note that we directly store the received power into the database without any calibration process.

In order to show the effectiveness of our approach, we compare the performance of our approach with some other range based approaches, such as, WLLS [8], LLS [7], 2WLLS [9], and the calibration approach [1]. It is worthy to point out that the estimated ranges of all the range based approaches are obtained from the calibrated range. i.e., we use the range estimates (8) instead of (5) as inputs for the different range based approaches.

In the online localization phase, we assume that the receiver locates at $(30,30,60)$. Then the real ranges between the receiver and the transmitters can be calculated using (5). Firstly, we compare the Mean Square Errors (MSEs) of different approaches when SNRs vary from $5 \mathrm{~dB}$ to $30 \mathrm{~dB}$ with equal spacing of $5 \mathrm{~dB}$. The SNR is defined as $10 \log 10^{1 / \sigma_{n}^{2}}$. We compute the RMSE base on 1000 independent runs. The RMSEs of the range based approaches and our proposed approach versus different SNRs are shown in Fig. 1. In Fig. 1, it is seen that the proposed fingerprinting based approach is superior to the other range based approaches in different SNR cases. Among the range based approaches, the calibrated approach has the worst performance because it is severely influenced by the estimated calibration factor $n$. Based on the same range estimate, 2WLLS gives a more accurate estimate than WLLS and LLS approaches.

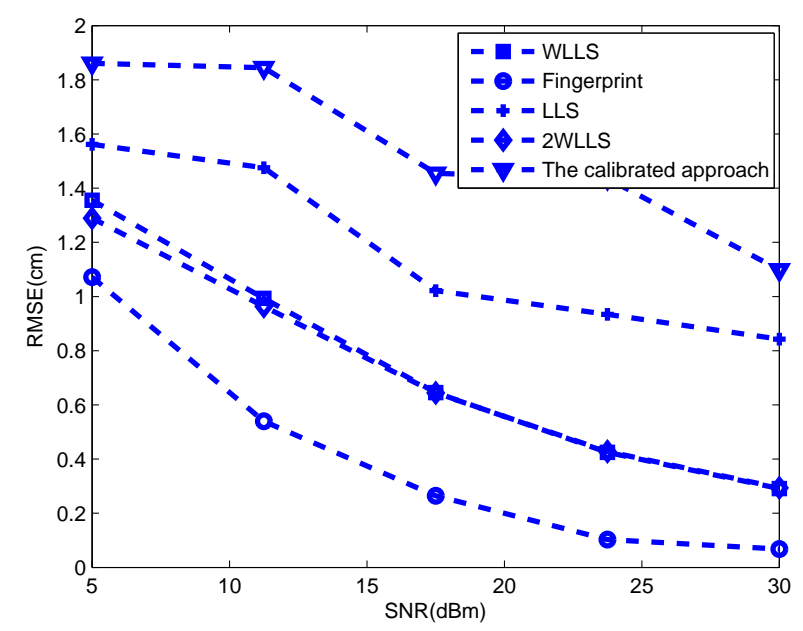

Fig. 1: Mean square root error (RMSE) comparison for different localization approaches.

\section{Conclusion}

We considered a LED localization problem in indoor environment. A fingerprint based approach is proposed to improve the accuracy of localization of LED receiver. Compared with some existing 
approaches, the proposed approach does not need any calibration process for range estimation. And the accuracy of LED localization can be further improved by selecting smaller grid distance. Unlike some existing range based approaches, the proposed approach is never affected by the errors from the range estimation. The main burden of our approach comes from building the radio map. Some low rank matrix completion algorithms and interpolation methods can be used to overcome the shortcoming, which will be researched in our next work.

\section{Acknowledgement}

This work was supported by the National Natural Science Foundation of China under Grant (No.61201277) and Non full-time graduate students teaching reform project of UESTC (Y03003023901002076).

\section{References}

[1] Hyun-Seung Kim, Deok-Rae Kim, Se-Hoon Yang, Yong-Hwan Son, and Sang-Kook Han, “An indoor visible light communication positioning system using a RF carrier allocation technique," Lightwave Technology, Journal of, vol. 31, no. 1, pp. 134--144, 2013.

[2] Thomas Q Wang, Y Ahmet Sekercioglu, Adrian Neild, and Jean Armstrong, "Position accuracy of time-of-arrival based ranging using visible light with application in indoor localization systems," Journal of Lightwave Technology, vol. 31, no. 20, pp. 3302--3308, 2013.

[3] Soo-Yong Jung, Swook Hann, and Chang-Soo Park, "TDOA-based optical wireless indoor localization using LED ceiling lamps," Consumer Electronics, IEEE Transactions on, vol. 57, no. 4, pp. 1592--1597, 2011.

[4] Muhammad Yasir, Siu-Wai Ho, and Badri N Vellambi, "Indoor positioning system using visible light and accelerometer," Journal of Lightwave Technology, vol. 32, no. 19, pp. 3306--3316, 2014.

[5] Gregary B Prince and Thomas DC Little, "A two phase hybrid RSS/AoA algorithm for indoor device localization using visible light," in Global Communications Conference (GLOBECOM), 2012 IEEE. IEEE, 2012, pp. 3347--3352.

[6] Anna Maria Vegni and Mauro Biagi, "An indoor localization algorithm in a small-cell LEDbased lighting system," in Proceedings of 3rd International Conference on Indoor Positioning and Indoor Navigation (IPIN 2012), 2012, pp. 1--7.

[7] Hing Cheung So and Lanxin Lin, "Linear least squares approach for accurate received signal strength based source localization," Signal Processing, IEEE Transactions on, vol. 59, no. 8, pp. 4035--4040, 2011.

[8] Lanxin Lin, HC So, Frankie KW Chan, YT Chan, and KC Ho, "A new constrained weighted least squares algorithm for TDOA-based localization," Signal Processing, vol. 93, no. 11, pp. 2872--2878, 2013.

[9] YT Chan and KC Ho, "A simple and efficient estimator for hyperbolic location," Signal Processing, IEEE Transactions on, vol. 42, no. 8, pp. 1905--1915, 1994. 